\title{
Neuritogenesis of Herbal (+)- and (-)-Syringaresinols Separated by Chiral HPLC in PC12h and Neuro2a Cells
}

\author{
Kenzo Chiba, ${ }^{*}, a$ Matsumi YamazaKI,${ }^{a}$ Emiko Umegaki, ${ }^{a}$ Ming Run Li, ${ }^{b}$ Zhen Wen Xu, ${ }^{b}$ \\ Sumio TeradA, ${ }^{c}$ Michihiro TAKA, ${ }^{c}$ Noriyuki NAOI,${ }^{d}$ and Tetsuro MoHrI ${ }^{a}$ \\ ${ }^{a}$ Department of Biodynamics, Faculty of Pharmaceutical Sciences, Hokuriku University; Ho-3 Kanagawa-machi, \\ Kanazawa, Ishikawa 920-1181, Japan: ${ }^{b}$ Tianjin Institute of Medical and Pharmaceutical Science; 96 Gui Zhou Road, \\ Heping District, Tianjin 300070, China: ${ }^{c}$ Natural Products Research Laboratory, Research Laboratory, Zenyaku Kogyo \\ Co., Ltd.; 2-33-7 Ohizumi-machi, Nerima-ku, Tokyo 178-0062, Japan: and ${ }^{d}$ Medical Devices Division, Kaneka \\ Corporation; 3-2-4 Nakanoshima, Kita-ku, Osaka 530-0005, Japan.
}

Received November 1, 2001; accepted March 4, 2002

Syringaresinol isolated from Epimedium koreanum NAKAI and Magnolia officinalis ReHD. et WILS. was subjected to optical resolution by chiral HPLC to give (+)- and (-)-enantiomers. The two syringaresinol enantiomers, as well as a mixture of their glucosides, showed dose-dependent neuritogenesis in a concentration range from 0.24 to $24 \mu_{M}$ in PC12h cells.

Key words syringaresinol; optical isomer; neuritogenesis; PC12h cell; Epimedium koreanum

We have carried out a continuing study of the neuron-stimulative substance in plants and found many lipophilic constituents that promote the differentiation (neurite outgrowth) of paraneurons, such as PC12h cells. ${ }^{1,2)}$ The lipophilic extracts of the aerial parts of Epimedium koreanum NAKAI or Epimedium macranthum MoRR. et DECNE. have been demonstrated to have neuritogenetic activity in $\mathrm{PC} 12 \mathrm{~h}$ cells and stimulate these cells excitedly to respond to carbachol. ${ }^{2)} \mathrm{We}$ have preliminarily found an active component of the $\mathrm{Et}_{2} \mathrm{O}$ extract of E. koreanum syringaresinol, which was found as itself or its glucosides in many of traditional Chinese drugs, including Acanthopanax senticosus HARMS $^{3)}$ and the bark of Eucommia ulmoides Oliv. ${ }^{4}$ It was previously shown to have a prominent effect on $\mathrm{PC} 12 \mathrm{~h}$ cells. ${ }^{5)}$ But the syringaresinol samples we isolated from the extract of E. koreanum seemed to be peculiarly racemic mixtures on the data of HPLC analysis with a chiral stationary phase. We report here the separation of optical isomers of syringaresinol from each of two species of plant, and the comparison of the biological activity of individual enantiomers and a glucoside mixture.

\section{MATERIALS AND METHODS}

The concentrated $\mathrm{Et}_{2} \mathrm{O}$ extract (35.2 g) of E. koreanum collected in northern China in July, 1998 was prepared at the Tianjin Institute of Medical and Pharmaceutical Science and chromatographed on a silica gel (Kieselgel 60) column by a successive elution with $\mathrm{CHCl}_{3}, \mathrm{CHCl}_{3}-\mathrm{MeOH}(3: 1)$ and $\mathrm{MeOH}$ (each 21). The evaporation residue $(17.5 \mathrm{~g})$ of the $\mathrm{CHCl}_{3}$ fraction was chromatographed on a silica gel column by a succcssive elution with $n$-hexane, $n$-hexane-EtOAc $(5: 1)$, benzene-EtOAc $(1: 1)$, benzene-EtOAc-MeOH $(1: 1$ : $0.1)$ and benzene-EtOAc-MeOH $(1: 1: 1)$. The most bioactive fraction from the benzene-EtOAc-MeOH $(1: 1: 0.1)$ eluate was chromatographed on TSK HW 40 (Tosoh, Japan) with an eluent, $\mathrm{MeCN}-\mathrm{H}_{2} \mathrm{O}(1: 1)$. The main fraction of the eluate was purified by Sephadex-LH20 chromatography with $\mathrm{MeOH}-\mathrm{H}_{2} \mathrm{O}(1: 1)$ to give syringaresinol $(3 \mathrm{mg})$, which was identified by comparison with UV, MS and NMR spectral data of an authentic sample.
Magnolia officinalis ReHD. et WILS., collected in China, was purchased from Matsuura Yakugyo Co., Ltd. (Nagoya, Japan). The voucher specimen (No 228110) was deposited in the natural product laboratory of the Research Laboratory, Zenyaku Kogyo Co., Ltd. Syringaresinol-4'-O- $\beta$-D-glucoside $(100 \mathrm{mg})$ isolated from the bark of M. officinalis according to the method of Yahara et al. ${ }^{6}$ was hydrolyzed by $\beta$-glucosidase (Sigma, U.S.A.) in $0.1 \mathrm{~m}$ acetate buffer (pH 5.0) at $37^{\circ} \mathrm{C}$ overnight. The reaction mixture was extracted three times with EtOAc to afford syringaresinol (31 mg). Each of the two racemic syringaresinol samples obtained from E. koreanum and M. officinalis was subjected to HPLC using a Chiralcel OD column (Daicel Chemical, Japan) and EtOH- $n$-hexane $(8: 2)$ as a mobile phase to give optically pure isomers (colorless needles).

Optical rotations were measured on a Perkin-Elmer 247 polarimeter. High resolution mass spectra were recorded on a JMS-SX102 MS instrument. NMR spectra were recorded on a JEOL EX270 NMR spectrometer. HPLC was performed on a Chiralcel OD (Daicel Chemical) column $(0.46 \times 25 \mathrm{~cm})$ using a JASCO TRI ROTAR-V PUMP with a UVIDEC-100$\mathrm{V}$ UV detector (eluent, EtOH- $n$-hexane $(8: 2)$ (flow rate, $0.5 \mathrm{ml} / \mathrm{min}$; detection, UV at $254 \mathrm{~nm}$ ).

$\mathrm{PC} 12 \mathrm{~h}$ cells were generously donated by Dr. Hatanaka, Osaka University, Japan, and cultured in Dulbecco's modified Eagle medium supplemented with precolostrum calf serum and heat-inactivated horse serum for growth, as described previously. ${ }^{7)}$ Neuro2a cells were purchased from the Institute for Fermentation, Osaka, Japan, and cultured in Eagle's minimum essential medium supplemented with non-essential amino acids and fetal bovine serum for growth. After $24 \mathrm{~h}$ of culture, the culture medium was replaced by serum-free medium to treat these cell lines with test compounds as specified in the results. Syringaresinol-4' $-O-\beta$-D-glucoside was dissolved in distilled water and ( + )- and $(-)$-syringaresinol in $75 \%$ dimethylsulfoxide before use. The neurite outgrowth of these cells was evaluated by measuring the length of the longest neurites of individual cells following $48 \mathrm{~h}$ of treatment with the test compounds as described previously. ${ }^{7)}$ One hundred cells in ten random fields in two culture dishes were 
averaged for each treatment.

\section{RESULTS AND DISCUSSION}

Optical Resolution by HPLC of Syringaresinol Syringaresinol samples isolated from $E$. koreanum and $M$. officinalis were identified by comparison with UV, MS and NMR spectral data of an authentic sample. Their optical rotation was estimated at almost naught, but varied from sample to sample. So they were then subjected to chiral chromatograhy (Fig. 1). On the basis of the results, the syringaresinol samples were supposed to be racemic mixtures.

Actually, identificatin of $(+)$ - and $(-)$-syringaresinol on the chromatograms was done by comparison with those of syringaresinol samples isolated from $M$. officinalis, because the amount was not enough to determine the optical rotation of separate enatiomers of samples from E. koreanum. The physical data of the enantiomers (in preceding and subsequent eluates, respectively) from $M$. officinalis are (+)-syringaresinol $\left([\alpha]_{\mathrm{D}}^{20}+53.3^{\circ}, c=0.36, \mathrm{CHCl}_{3} ; \mathrm{mp} 187.5-189.5\right.$ $\left.{ }^{\circ} \mathrm{C}\right)$ and $(-)$-syringaresinol $\left([\alpha]_{\mathrm{D}}^{20}-50.6^{\circ}, c=0.32, \mathrm{CHCl}_{3} ; \mathrm{mp}\right.$ $\left.188.5-190.5^{\circ} \mathrm{C}\right)$, occurring in the ratio of $c a .2: 1$ according

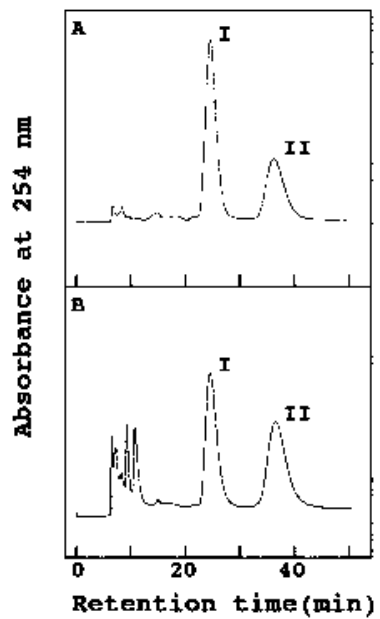

Fig. 1. Optical Resolution of Syringaresinol Samples by HPLC

The syringaresinol samples were applied to a Chiralcel OD column $(0.46 \times 25 \mathrm{~cm})$ and eluted isocratically with EtOH- $n$-hexane $(8: 2)$ at a flow rate of $0.5 \mathrm{ml} / \mathrm{min}$, with absorption monitored at $254 \mathrm{~nm}$. The elution profiles (A and B) represent syringaresinol samples isolated from E. koreanum and $M$. officinalis, respectively. I and II indicate (+)- and (-)-syringaresinol, respectively.

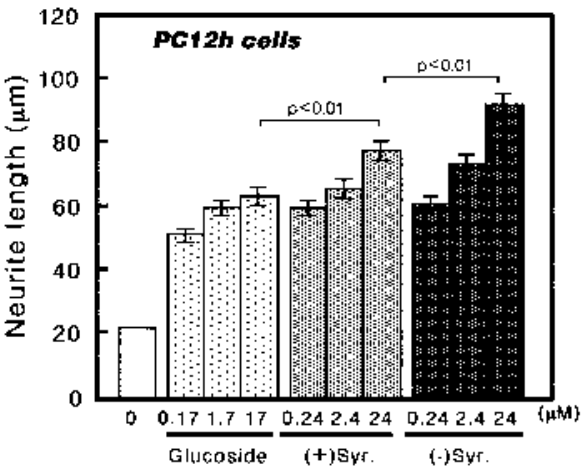

to the elution profile on chiral chromatography.

The Neuritogenic Activity of Optical Isomers of Syringaresinol in PC12h and Neuro2a Cells The neuritogenicity of $(+)$-syringaresinol obtained from $S$. baicalensis and (-)-syringaresinol from Scutellaria scandens BucH.HAM. ex D. Don was already reported with PC12h cells. ${ }^{5)}$ Here, we elaborate upon the effect of isolated enantiomers of syringaresinol as well as their glucoside mixture from the bark of M. officinalis on PC12h and Neuro2a cells. Consistent with our previous results, all of the glucosides and the two enantiomers were individually effective on the neurite outgrowth in both $\mathrm{PC} 12 \mathrm{~h}$ and Neuro2a cells (Fig. 2). At about $0.2 \mu \mathrm{M}$ of these compounds the effect was comparable between them in either cell line. But at higher doses (about 2 and $20 \mu \mathrm{M}$ ) of each, two enantiomers showed a generally stronger effect than the glucoside, especially, the $(-)$-isomer was discernibly more effective than any of the other compounds at a concentration of $24 \mu \mathrm{M}$ in either cell line. The neurite outgrowth activity of the mixture containing two enantiomers of syringaresinol was about similar in extention to the additive activity of each isomer (data not shown).

All of these results indicate that both $(+)$ - and (-)-syringaresinol, as well as a mixture of optical isomers of syringaresinol-4'-O- $\beta$-D-glucoside, are effective on neuritogenicity in cultured paraneurons, and that a racemic mixture of (+)- and (-)- syringaresinol would be responsible, at least partly, for the neurite outgrowth, i.e. cell differentiation or activation, of the paraneurons with the $\mathrm{Et}_{2} \mathrm{O}$ extract of $E$. $\mathrm{ko}$ reanum. It may be interesting to investigate the effect of these isomers on such activities of the central nervous system of animals as memory and behaviors, because the Chinese raw drug "Japanese name; Inyokaku" made of the dried herb of Epimedium species is an important element in many tonics, and is used in the treatment of aging-related amnesia and impotence. ${ }^{8)}$

Syringaresinol and its related compounds have several pharmacological activities including an inhibitory effect on cyclic AMP phosphodiesterase (PDE), ${ }^{9}$ a prolonging effect on the exercise time to exhaustion in chronic swiming stressed rat, ${ }^{10)}$ and an inhibitory effect on gastric ulcer. ${ }^{11)}$ The inhibitory activity on PDE of (+)-syringaresinol-di- $\beta$-glucoside and the enzymatic hydrolysate (syringaresinol) was considarable $\left(\mathrm{IC}_{50}: 12.5 \times 10^{-5} \mathrm{M}\right.$ and $17.5 \times 10^{-5} \mathrm{M}$, respectively), and the activity of $(-)$-syringaresinol-di- $\beta$-glucoside was

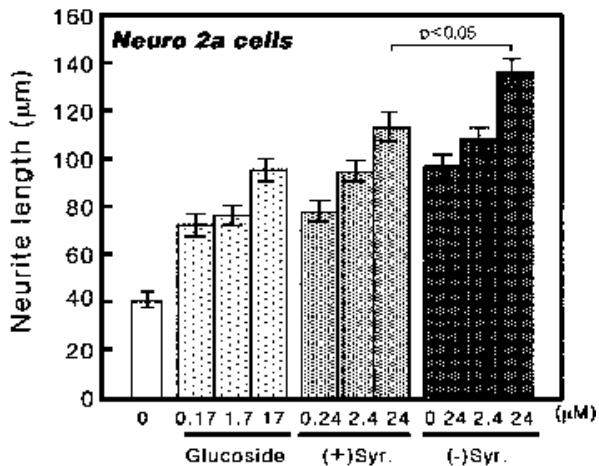

Fig. 2. The Neuritogenesis of Syringaresinol-4'-O- $\beta$-D-glucoside and (+)- and ( - -Syringaresinol in PC12h and Neuro2a Cells

Cells were cultured with or without (control) concentrations of test compounds as indicated for $2 \mathrm{~d}$. The neurite outgrowth was measured as described in Materials and Methods for 100 cells in each group and compared with the control, or between treated groups. The values are shown by the means \pm S.E.M. All of the means of treated groups are significantly $(p<0.01)$ large compared with the control. Statistical analysis was performed by ANOVA and Bonferroni's test. 
less $\left(\mathrm{IC}_{50}:>50 \times 10^{-5} \mathrm{M}\right)$, with specificity in the chiral isomers. Neuritogenesis was induced by cAMP in both PC12 and Neuro $2 \mathrm{a}$ cells used in the present study. ${ }^{12,13)}$ Therefore syringaresinol may be able to induce cAMP-dependent neuritogenesis through PDE inhibition. However, in contrast to the inhibitory activity on PDE mentioned above, the neuriogenic activity of the $(-)$ isomer was more effective than the $(+)$ isomer. Additional studies will be required to show the mechanism of syringaresinol.

Dual formation by a single enzyme of optically different bonds of specific groups is generally implausible in biology. It could be thought that the racemic formation of the respective bonds in the syringaresinol structure is performed nonenzymatically in those plants reported here. Such occurrence in both enantiomers of syringaresinol is suspected also in Acanthopanax senticosus (RUPR. et MAXIM.) HARMs (data not shown), which is useful as a source of tonic raw drug in Chinese traditional medicine. Syringaresinol in either form, even its glucosides, could be considered a key material in the search for neurotrophic and tonic drugs.

Acknowledgment This work was supported in part by grants of the special research fund of Hokuriku University.

\section{REFERENCES}

1) Mohri T., Chiba K., Yamazaki M., Shimizu M., Morita N., Planta Med., 58, 321-323 (1992).

2) Mohri T., Yamazaki M., Chiba K., Watanabe H., Shimizu M., J. Med. Pharmaceut. Soc. WAKAN-YAKU, 9, 182-189 (1992).

3) Nishibe S., Kinoshita H., Takeda H., Okano G., Chem. Pharm. Bull., 38, 1763-1765 (1990).

4) Deyama T., Chem. Pharm. Bull., 31, 2993-2997 (1983).

5) Yamazaki M., Hirota K., Chiba K., Mohri T., Biol. Pharm. Bull., 17, 1604-1608 (1994).

6) Yahara S., Nishiyori T., Kohda A., Nohara T., Nishioka I., Chem. Pharm. Bull., 39, 2024-2036 (1991).

7) Yamazaki M., Chiba K., Mohri T., Hatanada H., J. Neurochem., 79, 45-54 (2001).

8) Namba T., "The Encyclopedia of Wakan-Yaku (Traditional SinoJapanese Medicines) with Color Pictures," Vol. II, Hoikusha Publishing Co., Ltd., Osaka, 1994.

9) Nikaido T., Ohmoto T., Kinoshita T., Sankawa U., Nishibe S., Hisada S., Chem. Pharm. Bull., 29, 3586-3592 (1981).

10) Nishibe S., Kinoshita H., Takeda H., Okano G., Chem. Pharm. Bull., 38, 1763-1765 (1990).

11) Fujikawa T., Yamaguchi A., Morita I., Takeda H., Nishibe S., Biol. Pharm. Bull., 19, 1227-1230 (1996).

12) Okumura N., Miyatake Y., Takao T., Tamaru T., Nagai K., Okada M., Nakagawa H., J. Biochem. (Tokyo), 115, 304-308 (1994).

13) Mitui K., Tsuji S., Yamazaki M., Nagai Y., J. Neurochem., 57, 556561 (1991). 